\title{
Statistical Emulator Construction for Nonlinear Smart Systems
}

\author{
Francesca D. Reale ${ }^{1}$ and Ralph C. Smith ${ }^{2}$ \\ Center for Research in Scientific Computation \\ Department of Mathematics \\ North Carolina State University \\ Raleigh, NC 27695
}

\begin{abstract}
Comprehensive physical models can accurately quantify the dynamics of nonlinear and hysteretic systems but often require significant computational cost. This can reduce their effectiveness when performing sensitivity analysis, uncertainty analysis, parameter calibration or system design which typically requires multiple iterations of computationally expensive routines. This can also preclude the use of these models for real-time model-based control design. Emulators provide statistical approximations to comprehensive physical models which provide two advantages: high efficiency and statistical characterization of missing model components. We discuss the construction of statistical emulators to provide efficient surrogates for nonlinear smart material models. We will primarily focus on emulators for the homogenized energy model for ferroic compounds.
\end{abstract}

Keywords: Hysteresis, ferroelectric materials, nonlinear dynamics, statistical emulators

\section{Introduction}

Smart systems integrate sensors, actuators, and control laws to monitor and respond to external or internal stimuli. Ferroelectric materials are lightweight, low cost, and often can produce broadband responses. Their ease of use and functionality make ferroelectric materials ideal components of a number of smart systems [3]. For example, polyvinylidene fluoride (PVDF) is valuable in acoustic applications such as microphones [6]. High set point accuracy encourages the use of ferroelectric materials in nanopositioning as well. These materials are currently used as capacitors, transducers, and sensors. For example, barium titanate and lead titanate are relatively simple ferroelectric materials that are useful respectively in capacitors and transducers. However, in many applications these materials are increasingly being replaced by lead zirconate titanate (PZT) and lead magnesium niobate (PMN) [3]. For this reason, we focus on models that can characterize the behavior of PMN and PZT.

As a ferroelectric material cools below the Curie point (the phase transition temperature), it incurs a domain structure and spontaneous polarization that can be changed by an applied electric field and/or applied mechanical stress. The domain structure results from the alignment of dipoles that is energetically favorable (minimizes electrostatic and elastic energy) [3]. To use ferroelectric materials in the previously mentioned applications, one must characterize the response to applied field inputs. This includes the field-polarization (E-P) relationship.

In general, the relationship between the input field, stress and the output polarization, strain is nonlinear and hysteretic due to the noncentrosymmetric structure of ferroelectric materials. In low to moderate field levels, one can approximate the E-P relation using a linear model. However, in ferroelectric materials at high field levels, the nonlinear and hysteretic nature of the E-P relationship must be quantified. Furthermore, we desire a model that incorporates stress and temperature-dependence to characterize the behavior of, for example, PZT and PMN. The homogenized energy model incorporates the nonlinear constitutive relations, the lag effect between the inputs and outputs, and thermal relaxation [3]. The model is able to incorporate material nonhomogeneities via

\footnotetext{
${ }^{1}$ Email: fdreale@ncsu.edu, Telephone: (919) 515-3745

${ }^{2}$ Email: rsmith@eos.ncsu.edu, Telephone: (919) 515-7552
} 
the assumption that certain parameters are manifestations of underlying densities. We consider the computation cost of determining the polarization. We start with a fairly efficient code and have the goal of increasing its efficiency using an efficient surrogate of the polarization along with statistical quantification of missing model components. Later we can expand upon the technique to improve the efficiency of computationally expensive codes. An emulator provides such a statistical approximation of the polarization. To illustrate the technique, we introduce an example of a fairly simple emulator, the Kalman Filter.

We wish to use the Kalman filter as a simple emulator that can predict results. The Kalman filter is a recursive algorithm

$\begin{array}{lll}\text { Discrete Process } & & \\ & x_{k}=A x_{k-1}+B u_{k-1}+w_{k-1} & \text { (state) } \\ & z_{k}=H x_{k}+v_{k} & \text { (measurements) } \\ \text { Prediction } & \hat{x}_{k,-}=A x_{k-1}+B u_{k-1} & \text { (projects state) } \\ & v_{k,-}=A v_{k-1} A^{\mathrm{T}}+Q & \text { (projects covariance) } \\ \text { Correction } & & \\ & K_{k}=v_{k,-} H^{\mathrm{T}}\left(H v_{k,-} H^{\mathrm{T}}+R\right)^{-1} & \text { (updates Kalman gain) } \\ & \hat{x}_{k}=\hat{x}_{k,-}+K_{k}\left(z_{k}-H \hat{x}_{k,-}\right) & \text { (updates state) } \\ & v_{k}=\left(I-K_{k} H\right) v_{k,-} & \text { (updates covariance) }\end{array}$

that is used to estimate the state of a dynamic process governed by a linear stochastic difference equation. Let $w_{k} \sim N(0, Q)$ represent the process noise. Suppose we have measurements $z_{k}$ of the state. Let $v_{k} \sim N(0, R)$ represent the measurement noise $[2,5]$. These measurements are used to update the state estimate via feedback control in the form of a prediction step and a correction step. During the prediction step, we project the state and covariance ahead to obtain the prior estimate and prior estimate error covariance. During the correction step, the projected state is corrected using the measurements $z_{k}$ to obtain the posterior estimate and posterior estimate error covariance $[4,5]$. The discrepancy between the predicted measurements and the actual measurement is termed the residual. Observe that the updated state depends on the product of the residual and the gain. The Kalman gain is used as a "blending factor" to combine the residual with the projected state such that the posterior error covariance is minimized $[2,5]$. This combination leads to an updated, better estimate of the state [1]. Therefore we have an estimate with a prescribed statistical uncertainty associated with it that may be improved with each iteration.

\section{Homogenized Energy Model}

The homogenized energy model (HEM) incorporates the nonlinear and hysteretic constitutive relations, the lag effect between the input field and output polarization, and thermal relaxation. This model is able to characterize cases of nonhomogeneous materials, dipole switching, variable electric fields, and polycrystalline compounds. The material from this section can be found along with further detail in [3].

We consider the negligible thermal relaxation case first. The Gibbs energy

$$
G(E, P, T)=\psi(P, T)-E P
$$

is the difference between the Helmholtz energy

$$
\psi(P)=\left\{\begin{array}{l}
\frac{1}{2} \eta\left(P+P_{R}\right)^{2}, P \leq-P_{I} \\
\frac{1}{2} \eta\left(P-P_{R}\right)^{2}, P \geq P_{I} \\
\frac{1}{2} \eta\left(P_{I}-P_{R}\right)^{2}\left(\frac{P^{2}}{P_{I}}-P_{R}\right),|P|<P_{I}
\end{array}\right.
$$

and the work $E P$. Here $P_{R}$ and $P_{I}$ respectively denote the remanence and inflection polarization. The resulting average polarization kernel

$$
\bar{P}=\frac{1}{\eta} E+P_{R} \delta
$$


results from the equilibrium condition $\frac{\partial G}{\partial P}=0$. Here $\delta=+1$ for positively oriented dipoles and $\delta=-1$ for negatively oriented dipoles.

We also consider the possibility of thermal relaxation. The Boltzmann relation

$$
\mu(G)=C e^{-G V / k T}
$$

balances the Gibbs energy and the relative thermal energy $k T / V$ where $k$ is Boltzmann's constant and $\mathrm{V}$ is the reference volume. Consider a uniform lattice composed of $N=N_{+}+N_{-}$cells each with a positive or negative dipole orientation. The fraction of positively oriented dipoles $x_{+}=N_{+} / N$ and negatively oriented dipoles $x_{-}=N_{-} / N$ sum to unity. The average polarizations associated with positively and negatively oriented dipoles are respectively denoted by $\left\langle P_{+}\right\rangle$and $\left\langle P_{-}\right\rangle$and are given by

$$
\left\langle P_{+}\right\rangle=\int_{P_{0}(T)}^{\infty} P \mu(G) d P, \quad\left\langle P_{-}\right\rangle=\int_{-\infty}^{P_{0}(T)} P \mu(G) d P
$$

The local average polarization is

$$
\bar{P}=x_{+}\left\langle P_{+}\right\rangle+x_{-}\left\langle P_{-}\right\rangle .
$$

We denote the likelihoods of switching from negatively oriented to positively oriented and vice versa respectively by $p_{-+}$and $p_{+-}$. The evolution of dipole fractions is then given by

$$
\dot{x}_{+}=-p_{+-} x_{+}+p_{-+}\left(1-x_{+}\right)
$$

where the fraction $x_{-}$can be obtained using the equation $x_{-}+x_{+}=1$.

For a single crystal, the material properties are assumed to be homogeneous and isotropic which implies that the parameters are spatially invariant. However, this assumption is not valid for polycrystalline compounds which exhibit variable effective fields, anisotropy, and material nonhomogeneities. These nonhomogeneous effects are accounted for by assuming that parameters are manifestations of underlying densities.

We consider the interactive and coercive field parameters. The coercive field value $E_{C}=\eta\left(P_{R}-P_{I}\right)$ indicates when a dipole switch occurs. A dipole switch will occur at $-E_{C}$ and $+E_{C}$. The underlying density associated with $E_{C}$ is denoted by $\nu_{C}$. For physical considerations $\nu_{C} \geq 0, \nu_{C}$ is defined only for positive inputs, and $\left|\nu_{C}(x)\right| \leq c_{1} e^{-a_{1} x}$ for positive constants $a_{1}$ and $c_{1}$. Additionally, neighboring dipoles interact with the applied field to augment the applied field $E$. We denote this interaction field by $E_{I}$. The underlying density associated with $E_{I}$ is $\nu_{I}$. For physical considerations, $\nu_{I} \geq 0, \nu_{I}$ is an even function, and $\left|\nu_{I}(x)\right| \leq c_{2} e^{-a_{2}|x|}$ for positive constants $a_{2}$ and $c_{2}$. The densities are scaled such that

$$
\int_{0}^{\infty} \int_{-\infty}^{\infty} \nu\left(E_{C}, E_{I}\right) d E_{I} d E_{C}=C
$$

where $C$ is a scaling constant and $\nu\left(E_{C}, E_{I}\right)$ is the joint density.

The polarization model is formulated as the integral

$$
[P(E)](t)=\int_{0}^{\infty} \int_{-\infty}^{\infty} \nu_{C}\left(E_{C}\right) \nu_{I}\left(E_{I}\right)\left[\bar{P}\left(E+E_{I} ; E_{C}, \xi\right)\right](t) d E_{I} d E_{C}
$$

where $\xi$ is the initial dipole distribution.

We use numerical integration to approximate the value of the integral. Thus the integral is represented as the sum

$$
[P(E)](t)=\sum_{i=1}^{N_{i}} \sum_{j=1}^{N_{j}} \nu_{C}\left(E_{C_{i}}\right) \nu_{I}\left(E_{I_{j}}\right)\left[\bar{P}\left(E+E_{I_{j}} ; E_{C_{i}}, \xi_{j}\right)\right](t) v_{i} w_{j}
$$

where the abscissas $E_{I_{j}}, E_{C_{i}}$ and their respective weights $v_{i}, w_{j}$ are given by the chosen quadrature formula, e.g., see [3]. Four-point Gaussian quadrature is used to approximate the numerical value of the integral. 
To illustrate for the interaction field, the points $E_{I_{j}}$ and weights $w_{j}$ for the four-point truncated GaussLegendre quadrature rule used on each subinterval $\left[h_{q-1}, h_{q}\right]$ of $[-L, L]$ are

$$
\begin{aligned}
& E_{I_{q_{1}}}=h_{q-1}+h\left[\frac{1}{2}-\frac{\sqrt{15+2 \sqrt{30}}}{2 \sqrt{35}}\right], \quad w_{q_{1}}=\frac{49 h}{12(18+\sqrt{30})} \\
& E_{I_{q_{2}}}=h_{q-1}+h\left[\frac{1}{2}-\frac{\sqrt{15-2 \sqrt{30}}}{2 \sqrt{35}}\right], \quad w_{q_{2}}=\frac{49 h}{12(18-\sqrt{30})} \\
& E_{I_{q_{3}}}=h_{q-1}+h\left[\frac{1}{2}+\frac{\sqrt{15-2 \sqrt{30}}}{2 \sqrt{35}}\right], \quad w_{q_{3}}=\frac{49 h}{12(18-\sqrt{30})} \\
& E_{I_{q_{4}}}=h_{q-1}+h\left[\frac{1}{2}+\frac{\sqrt{15+2 \sqrt{30}}}{2 \sqrt{35}}\right], \quad w_{q_{4}}=\frac{49 h}{12(18+\sqrt{30})}
\end{aligned}
$$

where $h_{q}=-L+q h$ and $h=2 L / N_{q}$. The coercive field quadrature points and weights are defined similarly on $N_{p}$ subintervals. We note that throughout the analysis, we set the number of subintervals for the interactive field and coercive field densities to be equal so that $N_{q}=N_{p}$.

\section{Negligible Thermal Relaxation}

We first examine the outputs of the numerical implementation of the homogenized energy model under the assumption of negligible thermal relaxation. The relationship between the input field and output polarization is presented in Figure 1(a). We provide the polarization over a 73 second interval in Figure 1(b). The number of abscissas is chosen so that both the normal interactive field density and the lognormal coercive field density are sufficiently smooth. The field densities obtained when the number of subintervals $N_{q}=N_{p}=20$ are shown in Figure 1(c) and Figure 1(d). Note that there are 80 coercive field and 80 interactive field quadrature points. The computation time to obtain the approximation of the polarization (10) from four-point truncated Gauss-Legendre quadrature on $N_{p}=N_{q}=20$ subintervals is approximately 0.16 seconds. All future results are compared to this baseline approximation of (10). We run the computations to compute the baseline as well as subsequent approximations of the polarization on a Dell Optiplex 755 with an Intel(R) Core(TM)2 Quad CPU (a) $2.40 \mathrm{GHz}$ with $3 \mathrm{~GB}$ of RAM.

Using the Kalman Filter algorithm (1), we blend two approximations of the polarization integral (10) to obtain a better estimate. The goal is to reduce computation time while producing numerical results that accurately replicate the baseline results. We reduce computational time by reducing the number of quadrature points used in the approximation of the integral (10). We begin by reducing the number of intervals to reduce the number of abscissas. The number of quadrature points is reduced further by implementing a lower order Gaussian quadrature rule. Additionally, we consider a Newton-Cotes formula to exploit uniform abscissa spacing as a means of reducing computational time.

\subsection{Gaussian Quadrature}

We suggest a strategy to reduce computational time. We compute the polarization iteratively over a 73 second interval with time increments of $5 \times 10^{-2}$ seconds. This computation yields 1,461 polarization values. Let $P_{n, m}$ be the vector of 1,461 polarization values that result from using an $n$-point truncated Gauss-Legendre quadrature rule on $m$ intervals to approximate the value of the integral (10). Recall that the results of Figure 1 are obtained by utilizing the 4-point quadrature rule on $N=20$ intervals. We use $P_{4,20}$ as a baseline for comparison against other results. Consider two polarization vectors $P_{n_{1}, m_{1}}$ and $P_{n_{2}, m_{2}}$. Let $\sigma_{n, m}^{2}$ be the variance of the difference between $P_{n, m}$ and $P_{4,20}$ divided by the total number of quadrature points associated with calculating $P_{n, m}$. There are a total of $2 \times n \times m$ points involved in the calculation of $P_{n, m}$. Assume that $P_{n_{2}, m_{2}}$ is a more accurate calculation with respect to $P_{4,20}$ than $P_{n_{1}, m_{1}}$. Take $P_{n_{1}, m_{1}}$ to be the initial estimate of the polarization vector with uncertainty $\sigma_{n_{1}, m_{1}}^{2}$. Additionally, we have the "better" polarization estimate 


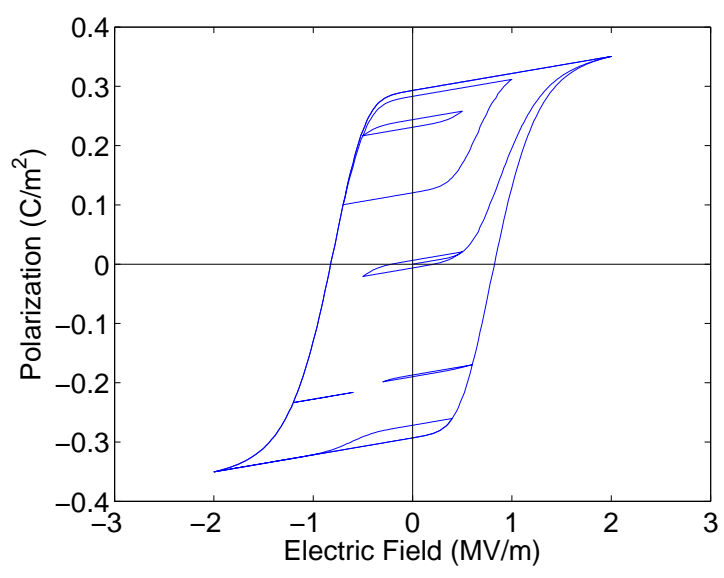

(a)

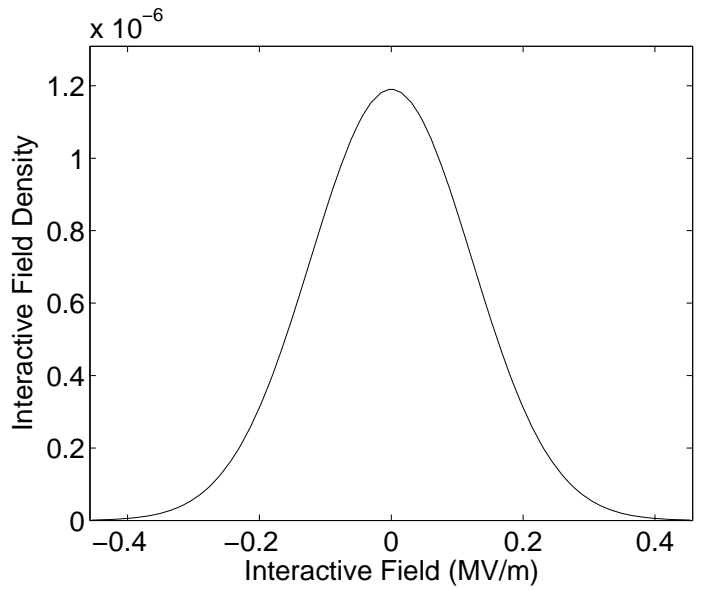

(c)

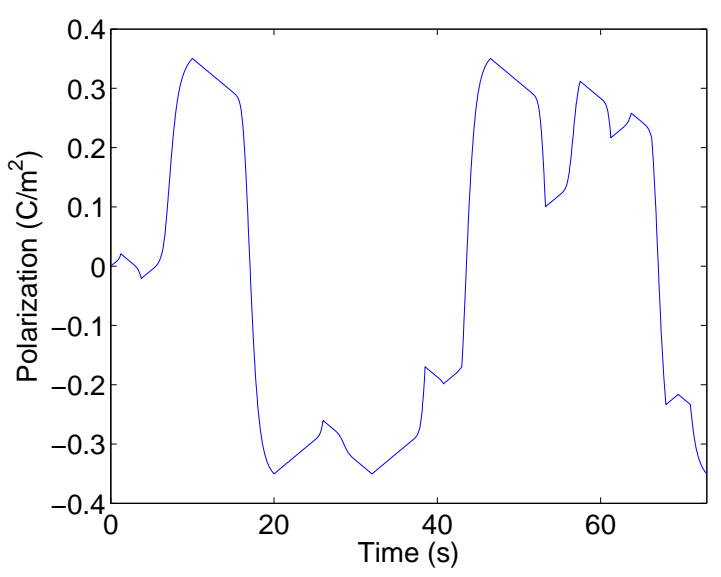

(b)

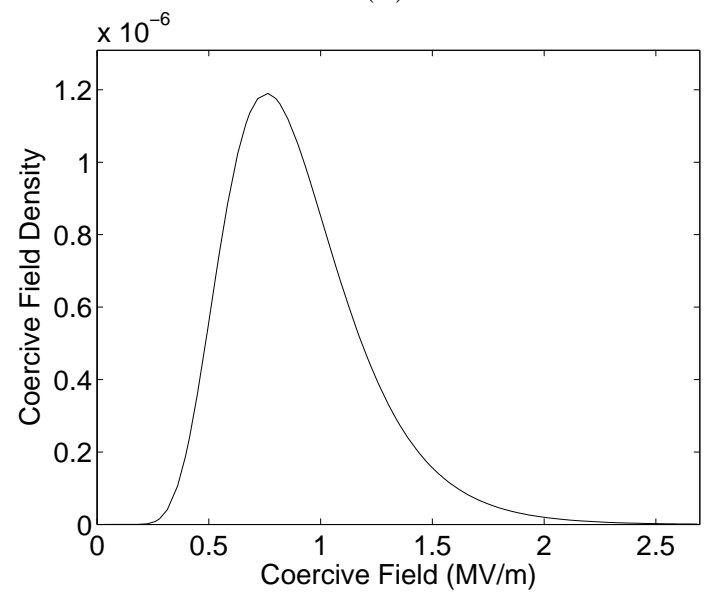

(d)

Figure 1: (a) Field-polarization relationship, (b) the polarization over a 73 second interval, (c) the interactive field density $\nu_{I}$, and (d) the coercive field density $\nu_{C}$.

$P_{n_{2}, m_{2}}$ with uncertainty $\sigma_{n_{2}, m_{2}}<\sigma_{n_{1}, m_{1}}$. Based on the Kalman Filter algorithm presented in (1), we propose that the estimate $\hat{x}^{(i)}$ of the $i^{t h}$ polarization vector component $P_{4,20}^{(i)}$ be given by

$$
\hat{x}^{(i)}=P_{n_{1}, m_{1}}^{(i)}+K_{1}\left(P_{n_{2}, m_{2}}^{(i)}-P_{n_{1}, m_{1}}^{(i)}\right)
$$

or, equivalently,

$$
\hat{x}^{(i)}=K_{2} P_{n_{1}, m_{1}}^{(i)}+K_{1} P_{n_{2}, m_{2}}^{(i)}
$$

where we compute the gain

$$
K_{i}=\frac{\sigma_{n_{i}, m_{i}}^{2}}{\sigma_{n_{1}, m_{1}}^{2}+\sigma_{n_{2}, m_{2}}^{2}}
$$

for $i=1, \ldots, 1461$. We may compute subsequent estimates of the polarization by updating the variance, gain, and polarization prediction as suggested by the Kalman Filter algorithm. This option will not be explored here, but will be considered in the future.

We begin by using the strategy suggested by the Kalman Filter to predict polarization values more efficiently by altering only the number of intervals $m$. The idea is to use two truncated Gauss-Legendre quadrature rules to obtain predictions and uncertainty estimates for the Kalman prediction strategy. We use the $P_{4,2}$ 
approximation and the $P_{4,4}$ approximation as separate "measurements." We compare both approximations to the $P_{4,20}$ approximation to obtain values of the uncertainty of these approximations

$$
\begin{aligned}
& \sigma_{4,2}^{2}=3.4781 \times 10^{-5} \\
& \sigma_{4,4}^{2}=7.5186 \times 10^{-7}
\end{aligned}
$$

which are used to compute the gains

$$
\begin{aligned}
& K_{1}=2.1160 \times 10^{-2} \\
& K_{2}=9.7884 \times 10^{-1} .
\end{aligned}
$$

Using these two approximations and their associated uncertainties, we find an estimate for each polarization value. This results in the approximation $\hat{x}_{G}$ of the polarization vector $P_{4,20}$. Figure 2 displays the comparison of $P_{4,20}$ and the approximation obtained with $P_{4,2}$ and $P_{4,4}$. Figure 2(a) presents the polarization $P_{4,20}$ whereas Figure 2(b) displays the approximation $\hat{x}_{G}$. The mean absolute difference between these two vectors of 1,461 values is $2.9205 \times 10^{-3}$. We also compare the time required to obtain both approximations. It takes around 0.16 seconds to obtain $P_{4,20}$ whereas it takes just over 0.03 seconds to obtain $\hat{x}_{G}$. The latter time includes the 0.02 seconds it takes to obtain $P_{4,4}$ and the 0.01 seconds to obtain $P_{4,2}$ as well as the time required to combine them as suggested in (14).

Figure 2 demonstrates that the approximation $\hat{x}_{G}$ does not appear as smooth as the $P_{4,20}$ polarization. While other smoothing techniques are considered, we choose to use moving average due to its simplicity and its efficiency.

The moving average technique averages $m_{a}$-many data points at a time to smooth out noisy, ordered data. We test the values $m_{a}=3,5,7$. We choose to average $m_{a}=5$ points at a time to smooth the approximation $\hat{x}_{G}$ and obtain a closer approximation to $P_{4,20}$. Let $\hat{x}_{G, S}^{(i)}$ be the $i^{\text {th }}$ component of the smoothed $\hat{x}_{G}$. Then the $i^{\text {th }}$ component of the approximation $\hat{x}_{G, S}$ is

$$
\hat{x}_{G, S}^{(i)}=\frac{1}{5} \sum_{n=i}^{4+i} \hat{x}_{G}^{(n)}
$$

for $i=1, \ldots, 1457$. It takes just over $10^{-3}$ seconds to run. Figure 3 presents the comparison between the baseline $P_{4,20}$ and the approximation $\hat{x}_{G, S}$. The resulting mean absolute difference from $P_{4,20}$ is $2.4257 \times 10^{-3}$.

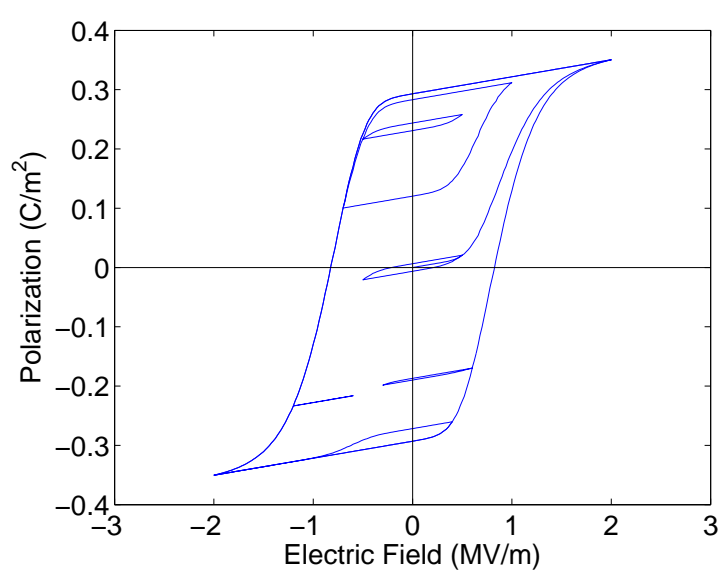

(a)

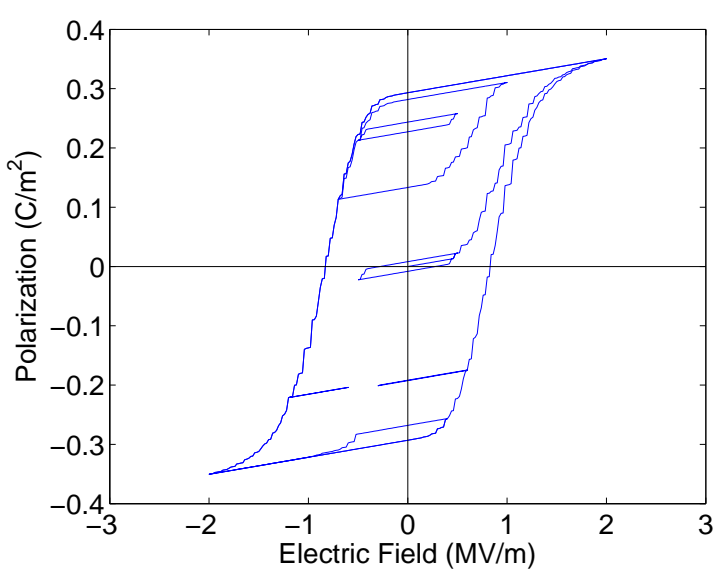

(b)

Figure 2: (a) Field versus the baseline approximation of the polarization $P_{4,20}$ and (b) field versus the polarization estimate $\hat{x}_{G}$. 


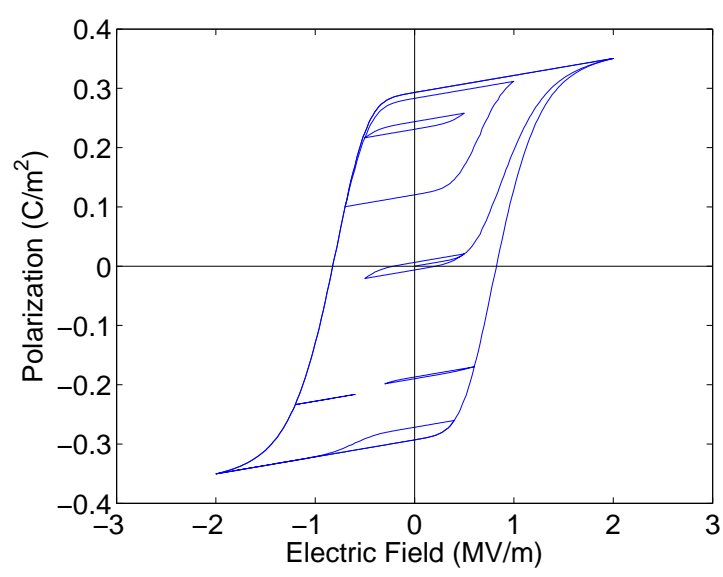

(a)

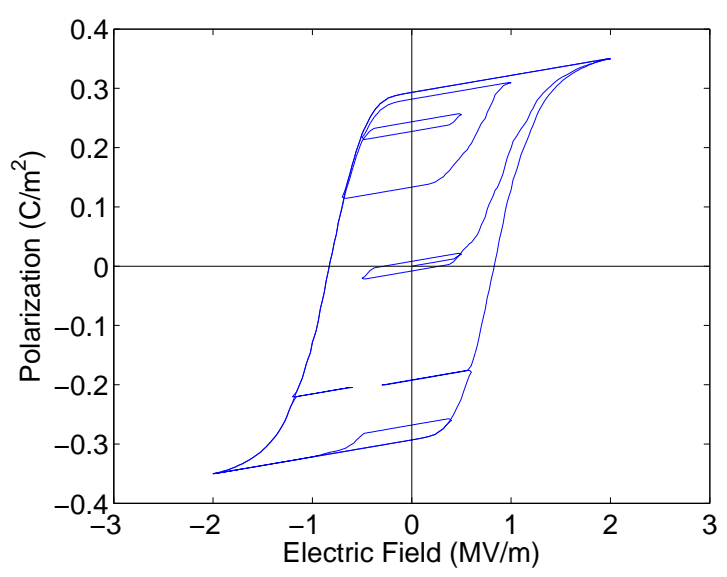

(b)

Figure 3: (a) Relationship between the field and the baseline approximation of the polarization $P_{4,20}$ and (b) field versus the approximation $\hat{x}_{G, S}$.

\subsection{Gaussian Quadrature: n-point Rules}

To reduce the computational time further without a significant increase in the mean absolute difference from $P_{4,20}$, we utilize the one-, two-, and three-point truncated Gauss-Legendre quadrature rules. The total number of abscissas used to approximate the integral (10) with $n$-point truncated Gauss-Legendre quadrature on $m$ subintervals is $2 \times n \times m$. Recall we set $N_{q}=N_{p}=m$.

To illustrate for the interaction field, we provide the points $E_{I_{j}}$ and weights $w_{j}$ for the $n$-point truncated Gauss-Legendre quadrature rule used on each subinterval $\left[h_{q-1}, h_{q}\right]$ of $[-L, L]$ in Table 1 where $h_{q}=-L+q h$ and $h=2 L / N_{q}$. The coercive field quadrature points and weights are defined similarly on $N_{p}$ subintervals.

The $P_{1,4}$ approximation and the $P_{3,4}$ approximation are used as separate "measurements." Both approxima-

\begin{tabular}{|l|l|l|l|}
\hline$n$ & $E_{I_{q_{i}}}$ & $w_{q_{i}}$ \\
\hline 1 & $E_{I_{q_{1}}}=h_{q-1}+\frac{h}{2}$, & $w_{q}=h$ \\
\hline 2 & $E_{I_{q_{1}}}=h_{q-1}+h\left[\frac{1}{2}-\frac{1}{2 \sqrt{3}}\right]$, & $w_{q_{1}}=\frac{h}{2}$ \\
& $E_{I_{q_{2}}}=h_{q-1}+h\left[\frac{1}{2}+\frac{1}{2 \sqrt{3}}\right]$, & $w_{q_{2}}=\frac{h}{2}$ \\
\hline 3 & $E_{I_{q_{1}}}=h_{q-1}+h\left[\frac{1}{2}-\frac{\sqrt{3}}{2 \sqrt{5}}\right]$, & $w_{q_{1}}=\frac{5 h}{18}$ \\
& $E_{I_{q_{2}}}=h_{q-1}+\frac{h}{2}$, & $w_{q_{2}}=\frac{8 h}{18}$ \\
& $E_{I_{q_{3}}}=h_{q-1}+h\left[\frac{1}{2}+\frac{\sqrt{3}}{2 \sqrt{5}}\right]$, & $w_{q_{3}}=\frac{5 h}{18}$ \\
\hline
\end{tabular}

Table 1: The $n$ quadrature points of the interval $\left[h_{q-1}, h_{q}\right]$ and their corresponding weights. 
tions are compared to the $P_{4,20}$ approximation to obtain measures of the uncertainty of these approximations

$$
\begin{aligned}
& \sigma_{1,4}^{2}=4.5902 \times 10^{-4} \\
& \sigma_{3,4}^{2}=1.9520 \times 10^{-6}
\end{aligned}
$$

which are used to compute the gains

$$
\begin{aligned}
& K_{1}=4.2346 \times 10^{-3} \\
& K_{3}=9.9577 \times 10^{-1} .
\end{aligned}
$$

We obtain $\hat{x}_{R}$ by blending the approximations $P_{1,4}$ and $P_{3,4}$. We compare the result to the baseline $P_{4,20}$ to find that the mean absolute difference between the two vectors is $4.6230 \times 10^{-3}$. It takes about 0.027 seconds to obtain $\hat{x}_{R}$ compared to 0.16 seconds to compute $P_{4,20}$. Figure 4 illustrates the comparison of the baseline $P_{4,20}$ to the approximation $\hat{x}_{R}$.

We apply moving average by averaging $m_{a}=5$ points at a time to smooth out $\hat{x}_{R}$. The smoothing takes just over one-thousandth of a second and yields a mean absolute difference of $3.9425 \times 10^{-3}$. Let $\hat{x}_{R, S}$ denote the smoothed $\hat{x}_{R}$ approximation. Figure 5 presents the comparison of the baseline $P_{4,20}$ to the approximation $\hat{x}_{R, S}$.

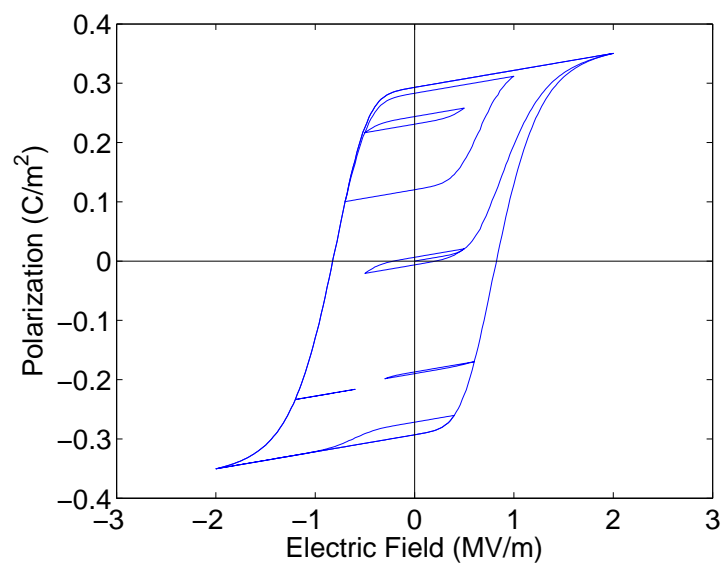

(a)

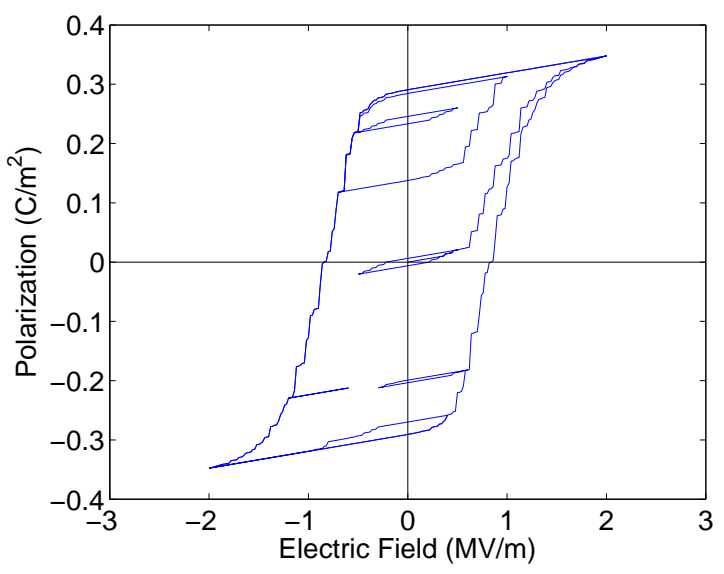

(b)

Figure 4: (a) Hysteretic relationship between the field and the baseline approximation of the polarization $P_{4,20}$ and (b) field versus the polarization estimate $\hat{x}_{R}$.

\subsection{Trapezoid Rule}

The approximations in Subsections 3.1 and 3.2 utilize Gaussian quadrature rules. Now the uniform abscissa spacing of Newton-Cotes quadrature rules is exploited to further reduce computational time. We illustrate the trapezoid rule for the interactive field. Consider a trapezoid rule approximation of the integral over $[-L, L]$. Let $N_{t}$ be the number of quadrature points. The Newton-Cotes rules use uniformly spaced quadrature points $x_{k}=-L+k h$ where $h=\frac{2 L}{N_{t}-1}$ is the stepsize and $k=0,1, \ldots, N_{t}-1$. Let $y_{k}$ be the value of the integrand at $x_{k}$. Then the integral is approximated by $h\left(\frac{1}{2} y_{0}+y_{1}+\ldots+y_{N_{t}-2}+\frac{1}{2} y_{N_{t}-1}\right)$. The trapezoid rule for the coercive field is defined similarly. Observe that a benefit of using uniformly spaced quadrature points is that one can reuse some abscissas as one increases the number of abscissas. So one may reuse previous calculations resulting in less computational cost. Let us call $P_{N_{t}}$ the vector of 1,461 polarization values obtained when the trapezoid rule with $N_{t}$ abscissas is used. We avoid calculation of the logarithm of zero by setting any zero quadrature point to machine epsilon $2.2 \times 10^{-16}$.

We use the strategy suggested in (14) to predict polarization values. The idea is to use two trapezoid rules with different numbers of abscissas to obtain approximations and uncertainty estimates for the Kalman 


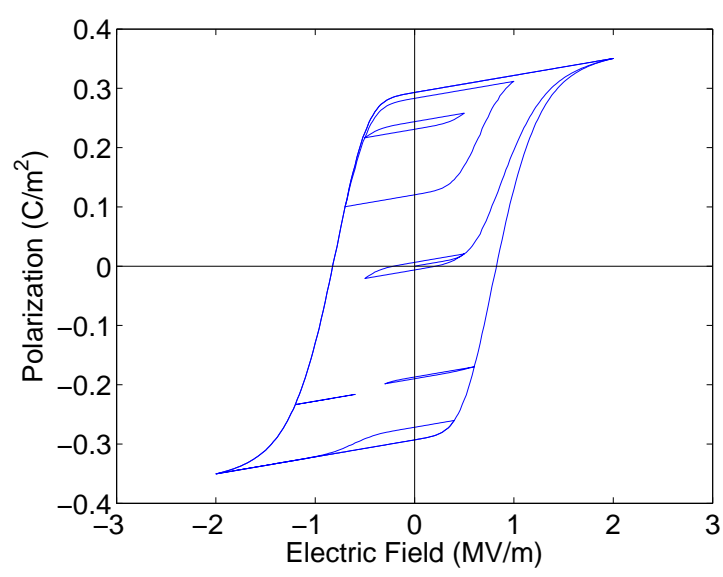

(a)

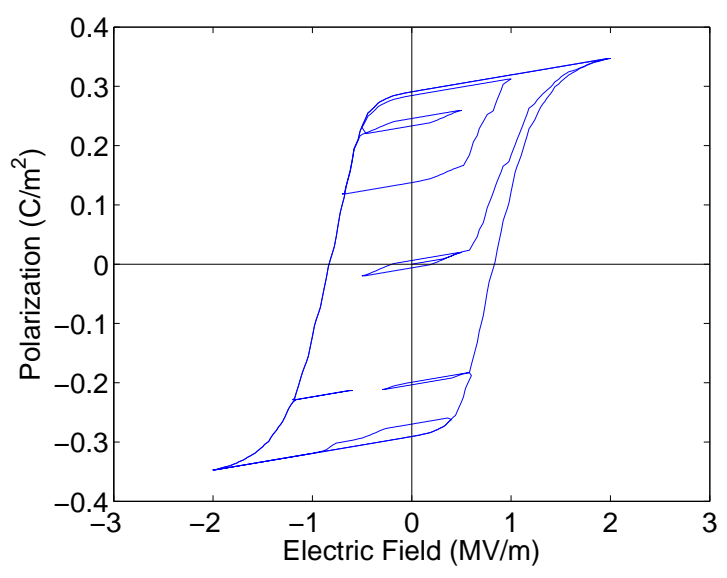

(b)

Figure 5: (a) Relationship between the field and the baseline approximation of the polarization $P_{4,20}$ and (b) field versus the approximation $\hat{x}_{R, S}$.

prediction strategy. As noted earlier, the components of the vector $P_{4,20}$ are assumed to be the true polarization values.

We wish to find the number of trapezoid rule points necessary to compute polarization values comparable to the $P_{4,20}$ values. We test numerous values of $N_{t}$. We visually and numerically compare $P_{N_{t}}$ to $P_{4,20}$ for varying values of $N_{t}$. The mean absolute difference from the $P_{4,20}$ vector of polarization values is on the order of $10^{-4}$ for each vector. The results are displayed in Table 2 and in Figure 6 . Note the time required to obtain the polarization vector $P_{4,20}$ is about 0.16 seconds. We note that the polarization computed when $N_{t}=72$ abscissas are used appears sufficiently accurate.

Similar to what is presented in (14), we propose that the $i^{t h}$ prediction $\hat{x}^{(i)}$ of the polarization be given by

$$
\hat{x}^{(i)}=K_{2} P_{N_{t_{1}}}^{(i)}+K_{1} P_{N_{t_{2}}}^{(i)}
$$

where we compute the gain

$$
K_{i}=\frac{\sigma_{N_{t_{i}}}^{2}}{\sigma_{N_{t_{1}}}^{2}+\sigma_{N_{t_{2}}}^{2}}
$$

for $i=1,2, \ldots, 1461$.

We use the $P_{4}$ approximation and the $P_{9}$ approximation as separate "measurements." It takes about 0.024 seconds to blend the two approximations to obtain the Kalman approximation $\hat{x}_{T}$. We compare the result to the $P_{4,20}$ approximation and find a mean absolute difference of $8.7363 \times 10^{-3}$. We smooth this approximation to obtain the estimate $\hat{x}_{T, S}$. The smoothing step takes one-thousandth of a second. The mean absolute difference between the approximation $\hat{x}_{T, S}$ and the baseline $P_{4,20}$ is $7.6445 \times 10^{-3}$. The visual comparison to the approximation $P_{4,20}$ presented in Figure 7 .

\begin{tabular}{|l|l|l|}
\hline$N_{t}$ & $\operatorname{mean}\left(\left|P_{4,20}-P_{N_{t}}\right|\right)$ & Time $(\mathrm{s})$ \\
\hline 48 & $9.5595 \times 10^{-4}$ & 0.062 \\
72 & $4.9040 \times 10^{-4}$ & 0.13 \\
128 & $2.7593 \times 10^{-4}$ & 0.38 \\
\hline
\end{tabular}

Table 2: Numerical and chronological comparison of trapezoid rule approximation $P_{N_{t}}$ of (10) to the baseline approximation $P_{4,20}$. The computational time corresponding to the baseline approximation is approximately 0.16 seconds. 


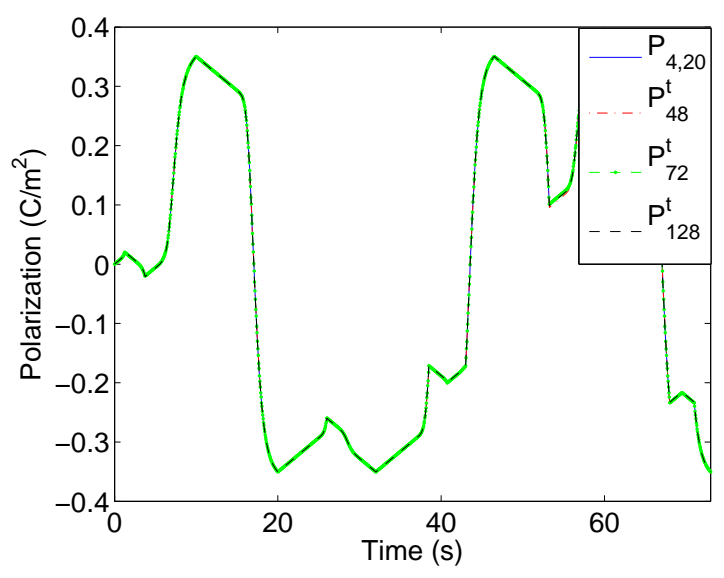

(a)

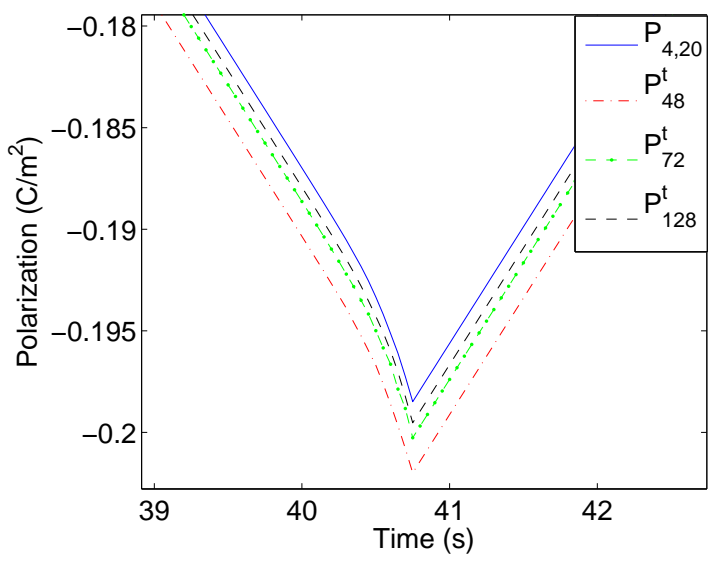

(b)

Figure 6: (a) Comparison of the trapezoid rule approximations $P_{N_{t}}$ to the baseline approximation $P_{4,20}$ over a 73 second interval and (b) expanded view of the approximations.

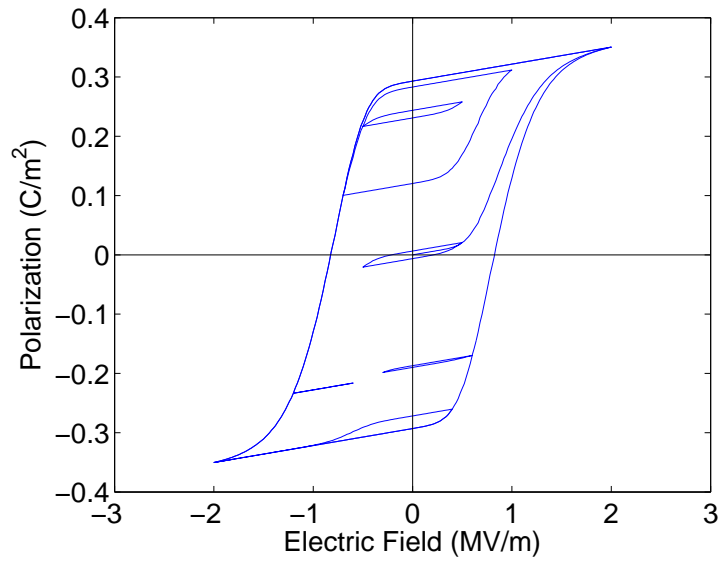

(a)

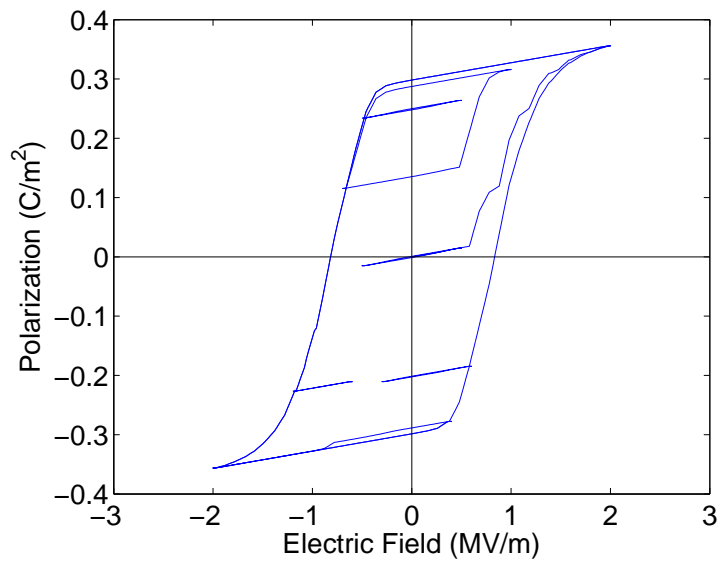

(b)

Figure 7: (a) Relationship between the field and the baseline approximation of the polarization $P_{4,20}$ and (b) field versus the approximation $\hat{x}_{T, S}$.

\subsection{Summary of Negligible Thermal Relaxation Results}

A summary of the results from Section 3 is presented in Table 3. We observe that the computational cost of all three Kalman Filter estimates is the same to one significant figure. The approximation $\hat{x}_{G, S}$ corresponds to the least mean absolute difference from the baseline approximation $P_{4,20}$. Thus the preferred strategy is to obtain two four-point Gaussian quadrature rule approximations on $m \leq 20$ intervals and blend them using the Kalman Filter algorithm as proposed in (14).

\section{Thermal Relaxation}

The homogenized energy model is able to incorporate thermal relaxation. Section 3 covers the details of the negligible thermal relaxation case. When thermal relaxation is incorporated, the calculations are significantly slower.

Let $R_{n, m}$ be the vector of 1,461 polarization values that result from using an $n$-point quadrature on $m$ intervals when thermal relaxation is incorporated. The relationship between the field and the polarization calculation 


\begin{tabular}{|l|l|l|}
\hline Approximation $\hat{x}$ & $\operatorname{mean}\left(\left|P_{4,20}-\hat{x}\right|\right)$ & Time $(\mathrm{s})$ \\
\hline$P_{4,20}$ & 0 & 0.16 \\
$\hat{x}_{G, S}$ & $2.4257 \times 10^{-3}$ & 0.031 \\
$\hat{x}_{R, S}$ & $3.9425 \times 10^{-3}$ & 0.028 \\
$\hat{x}_{T, S}$ & $7.6445 \times 10^{-3}$ & 0.025 \\
\hline
\end{tabular}

Table 3: Numerical and chronological comparison of approximations to the baseline approximation $P_{4,20}$.

$R_{4,20}$ is presented in Figure 8(a). We use $R_{4,20}$ as a baseline for comparison against other approximations of the polarization.

We blend the two measurements $R_{4,2}$ and $R_{4,4}$ using the Kalman filter strategy suggested by (14). This yields the vector of polarization values $\hat{x}_{T R}$. It takes about 1.14 seconds to obtain the baseline $R_{4,20}$. We blend $R_{4,2}$ and $R_{4,4}$ to obtain the smoothed approximation $\hat{x}_{T R}$ in 0.13 seconds. The mean absolute difference from $R_{4,20}$ is $1.8190 \times 10^{-3}$. Figure $8(\mathrm{~b})$ displays the field versus the smoothed polarization $\hat{x}_{T R}$.

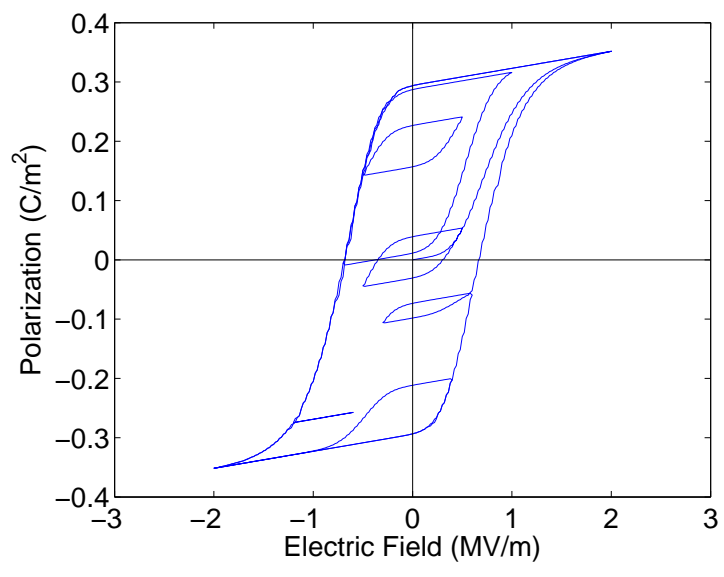

(a)

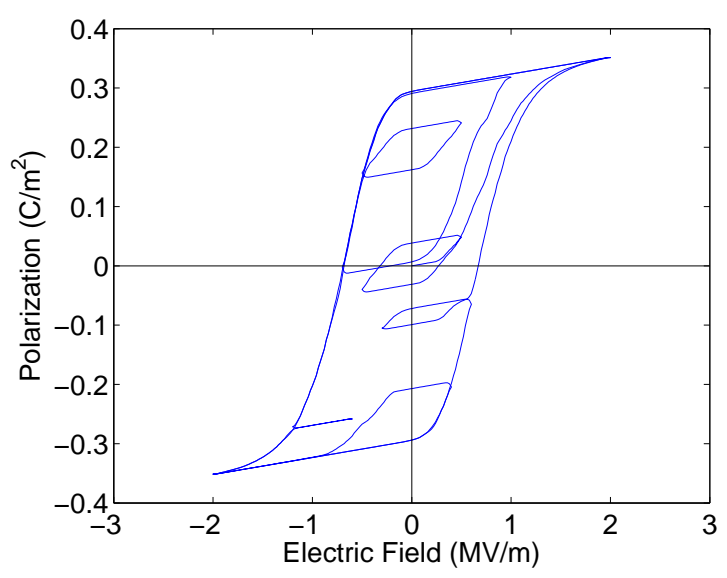

(b)

Figure 8: (a) Relationship between the field and the baseline approximation of the polarization $R_{4,20}$ and (b) the field versus the approximation $\hat{x}_{T R}$ smoothed with moving average.

\section{Concluding remarks}

The results of this investigation demonstrate that the Kalman filter works well as an emulator for approximating polarization in the homogenized energy model. We achieve a greater than $80 \%$ reduction in computation time while consistently maintaining a mean absolute difference from baseline of less than $10^{-2}$. This was accomplished using one iteration of the Kalman Filter strategy. Consideration of multiple iterations of the Kalman Filter strategy should be beneficial in reducing the mean absolute error at the cost of a minor time increase for subsequent iterations and updated estimates of the state, variance, and gain. Other emulators may provide additional benefits and will be considered in future work.

\section{Acknowledgments}

This research was supported in part by the Air Force Office of Scientific Research through the grants AFOSR-FA9550-04-1-0203 and AFOSR-FA9550-08-1-0348. 


\section{References}

[1] Gershenfeld, N., The Nature of Mathematical Modeling, Cambridge, Cambridge, 1999.

[2] Grewal, M.S. and Andrew, A.P., Kalman Filtering: Theory and Practice Using MATLAB, John Wiley and Sons, Hoboken, NJ, 2008.

[3] Smith, R.C., Smart Material Systems: Model Development, SIAM, Philadelphia, 2005.

[4] Stengel, R. F., Optimal Control and Estimation, Dover, New York, 1994.

[5] Welch, G. and Bishop,G., An Introduction to the Kalman Filter, University of North Carolina, Department of Computer Science, TR 95-041, July 24, 2006 < http://www.cs.unc.edu/ welch/kalman/kalmanIntro.html>.

[6] Xu, Y., Ferroelectric Materials and their Applications, North-Holland, Amsterdam, 1991. 